\section{P.1.29 COMMUNICATION AND DISSEMINATION IN THE OMEGA-NET COST ACTION}

${ }^{1}$ Damien McElvenny*, ${ }^{2}$ Michelle Turner, ${ }^{3}$ Alex Burdorf, ${ }^{4}$ Neil Pearce, ${ }^{5}$ Ingrid Mehlum. ${ }^{1}$ Institute of Occupational Medicine, Edinburgh, UK; ${ }^{2}$ Barcelona Institute for Global Health (IS Global), Barcelona, Spain; ${ }^{3}$ Erasmus University, Rotterdam, The Netherlands; ${ }^{4}$ London School of Hygiene and Tropical Medicine, London, UK; ${ }^{5}$ 5STAMI (National Institute of Occupational Health), Oslo, Norway

\subsection{6/OEM-2019-EPI.230}

OMEGA-NET (the Network on the Coordination and Harmonisation of European Occupational Cohorts - http:// omeganetcohorts.eu/) is a 4 year EU-funded COST Action that commenced in November 2017. The overarching concept of OMEGA-NET is to create a network to optimise and integrate occupational, industrial, and population cohorts at the European level, and to provide a foundation for an enhanced evidence base for the identification of health risks and gains related to occupation and employment to foster safe and healthy preventive strategies and policies.

OMEGA-NET has research and capacity-building objectives, including:

- Coordinate and integrate cohorts on occupational health in Europe

- Implement an online interactive tool with detailed information on existing cohorts

- Facilitate work on harmonisation of occupational exposure and standardisation of health outcome information and new protocols for data collection

- Connect scientific communities on occupational health in Europe

- Provide networking and leadership opportunities for early career researchers, as well as researchers from COST Inclusiveness Target Countries

- Provide training in occupational epidemiology and exposure assessment

- Our stakeholders are at National, European (e.g. EU, EUOSHA) and International levels (e.g. WHO, ILO). Scientists are under pressure to promote their research and the reasons for promoting OMEGA-NET include:

- Attracting future research funding in occupational health;

- Building new connections outside our Action, promoting research in other countries; and

- Putting occupational epidemiology on the agenda of EU and international (and national) policy-makers

We will present our Science Communication and Dissemination Plan, which sets out how we are interacting with and influencing key high-level stakeholders, and what we hope to achieve. We hope OMEGA-NET will inform occupational health research priority setting and promote a lively discussion on communication of research in occupational health.

\section{P.1.30 ASSESSMENT OF SICK BUILDING SYNDROME AMONG EMPLOYEES IN THE COMMERCIAL CENTERS IN NIŠ, SERBIA}

${ }^{1}$ Bojana Miladinovic*, 'Maja Nikolic, ${ }^{1}$ Aleksandra Stankovic, ${ }^{2}$ Sci Visa Tasic. ${ }^{1}$ Faculty of Medicine University of Nis, Nis, Serbia; ${ }^{2}$ Mining and Metallurgy Institute, Bor, Serbia

10.1136/OEM-2019-EPI.231
Sick Building Syndrome (SBS) is a diseases associated with indoor air quality accompanied with various nonspecific symptoms that occur in the occupants of a building. This syndrome has been the subject of serious scientific investigation in the past years, but there are not enough studies in transition countries.

The aim of this study was to investigate the symptoms of the syndrome among employees in the commercial centers in Niš, Serbia.

Methods The cross-sectional study was conducted amongst employees of two commercial centers in the city of Niš, Serbia. In this study the MM-040EA questionnaire was used with two additional questions and 1152 employed were interviewed during the period of three years. Data extracted from the questionnaires were analyzed using the chi-square test and binary logistic regression.

Results The prevalence of SBS was high. The most common symptoms reported by employees included high room temperature $(74.9 \%)$, stuffy air $(73.5 \%)$, and dry air $(75.7 \%)$, while rare complaints were towards static electricity $(47.3 \%)$ and low room temperature (45.2\%). Binary logistic regression showed that too low room temperature $(\mathrm{p}=0.002)$, dry air $(p=0.015)$, static electricity $(p=0.007)$ and noise $(p=0.024)$ were the most important factors for the high symptoms score. A relatively small number of sick absence (13.4\%) was found among subjects working in the investigated commercial centers.

Conclusion The high prevalence of SBS symptoms in the environment of commercial centers was almost associated with factors of unpleasant microclimate. So improvement of environmental conditions such as increasing the efficiency of the ventilation system, increasing fresh air flow in the sector and noise prevention, as well as enhancing the quality of working life will motivate the employees and increase productivity in the workplace. The occupational health care workers play an important role in educating of workers and their employers.

\section{P.1.31 LIMIT VALUES FOR METALS: DISCREPANCIES BETWEEN DERIVATION FROM ANIMAL EXPERIMENTS AND EPIDEMIOLOGICAL STUDIES/WORKPLACE OBSERVATIONS WITHOUT EFFECTS AT HIGHER EXPOSURES}

Dirk Pallapies*, Peter Welge, Thomas Bruening. Institute for Prevention and Occupational Medicine of the German Social Accident Insurance, Bochum, Germany

\subsection{6/OEM-2019-EPI.232}

Risk assessment/limit value setting for metals at the workplace is often based primarily on animal data. Epidemiological data providing information on quantitative exposureresponse relationships is rarely available, but should be used preferentially if of sufficient quality, as in the case of chromium(VI). In Germany, the Committee of Hazardous Substances has derived an assessment criterion for chromium (VI) of $1 \mu \mathrm{g} / \mathrm{m}^{3}$ reflecting a tolerable risk of 4 additional cancer cases in 1000 workers exposed over their whole working life. For various metals human data is less informative. Thus, for cobalt, a tolerable concentration of $5 \mu \mathrm{g} / \mathrm{m}^{3}$ (respirable fraction) as been calculated based on inhalation studies in rats and mice which developed lung tumors after exposure to more than $1 \mathrm{mg} / \mathrm{m}^{3}$. However, recently 
published epidemiological studies among more than 30000 hardmetal and cobalt production workers do not provide any evidence for carcinogenicity in humans at exposures in the range from $10-100 \mu \mathrm{g} / \mathrm{m}^{3}$ - between the tolerable concentration based on animal data and the concentration used in the animal experiments. The implications for risk assessment will be discussed also taking into account additional epidemiological data addressing potential inflammatory or fibrogenic effects with impairment of lung function at higher workplace exposures. Copper is another compound for which in Germany a limit value has been proposed by the MAK (maximal workplace concentration) commission based on animal data. Based on a 28 day rat inhalation study with copper(I) oxide which demonstrated inflammatory effects at concentrations starting around $200 \mu \mathrm{g} / \mathrm{m}^{3}$ a MAK value of $10 \mu \mathrm{g} / \mathrm{m}^{3}$ (respirable fraction) has been derived. However, copper has been used at workplaces for centuries at concentrations even in the $\mathrm{mg}$ range without observation of clear health effects. Potential explanations for these discrepancies as well as requirements of additional health data will be presented.

\section{P.1.32 COMPARISONS OF THE RESULTS FROM ERGONOMIC TOOLS BETWEEN RAPID ENTIRE BODY ASSESSMENT AND QUICK EXPOSURE CHECK IN A STEEL FACTORY} ${ }^{1,2}$ Khongrit Pinyowiwat*, ${ }^{1}$ Soontorn Supapong, ${ }^{1}$ Thanapoom Rattananupong. ${ }^{1}$ Faculty of
Medicine, Chulalongkorn University, Bangkok, Thailand; ${ }^{2}$ Occupational and Environmental
Institute of Nopparat Rajathanee Hospital, Bangkok, Thailand

\subsection{6/OEM-2019-EPI.233}

Conducting ergonomic risk assessment is important as it helps employers identify the significant risks in their workplace and will resulting in adequate preventive measures. Simpler observational techniques is one of the types of ergonomic evaluation tools which is commonly used. It is practical, user-friendly and gathering information from both employees and practitioners. This paper presents comparisons between two observational ergonomics tools for determining ergonomics risk factors for work-related musculoskeletal disorders. The Rapid Entire Body Assessment (REBA) and Quick Exposure Check (QEC) were used to assess 296 employees participating in the study from all 12 sections which involved different work tasks in a steel factory. The results are compared using three risk categories (low, moderate, high). Standardised Nordic questionnaires was also used for evaluating the musculoskeletal disorders and effects on working. Data were gathered using questionnaires and evaluations taken at workstation. The findings show the results analyzed using weighted kappa statistic and prevalence of musculoskeletal disorders among workers.

\section{P.1.36 OCCUPATIONAL COHORT STUDIES: SAFEGUARDING A VALUABLE RESOURCE}

${ }^{1}$ Katherine Venables*, ${ }^{2}$ Nicola Fear, 'Lucy Carpenter, ${ }^{3}$ Thomas Keegan, ${ }^{1}$ Claire Brooks, ${ }^{2}$ Gemma Archer. ${ }^{1}$ University Of Oxford, Oxford, UK; ${ }^{2}$ King's College London, London, UK; ${ }^{3}$ Lancaster University, Lancaster, UK

10.1136/OEM-2019-EPI.234
An occupational cohort study is the most robust epidemiological design for studying the effects of workplace hazards and the findings can be extended to the general environment. A cohort may be time-consuming, expensive, and labour-intensive to set up but, once done, it can be extended forward in time, as well as laterally to incorporate new outcome variables, and it can also support nested case-control studies. It is therefore important that the human and material investment is preserved so that these valuable resources can be fully exploited.

In recent years, the bureaucratic burden on researchers in many countries has increased. In the UK, for example, research ethics, data protection, and data access application procedures have become more cumbersome, with an increase in the number of supporting documents required from researchers. Although fast-track procedures exist, epidemiological studies often require the same formal procedures and oversights as more invasive and potentially dangerous physiological and pharmacological studies.

Fortunately, there are now initiatives which support occupational cohort studies. The UK Medical Research Council (MRC), for example, published in 2014 a review and guidance about maximising the value of UK population cohorts and it has also set up a Cohort Strategic Review Group to pre-assess funding applications for new cohorts and for updates to existing cohorts (http://mrc.ukri.org). As another example, OMEGA-NET has been set up to 'create a network to optimize and integrate occupational, industrial, and population cohorts at the European level' (http://omeganetcohorts.eu/).

We propose that a checklist be defined for assessment of research protocols for new cohorts or updates to existing cohorts, in order to assist official committees in their work and streamline the approval process for both researchers and committees. EPICOH would be wellplaced to draft and promulgate such a checklist, working with interested organisations, such as OMEGA-NET and the UK MRC.

\section{P.1.37 A STUDY OF THE INCIDENCE RATE AND RISK FACTORS OF METABOLIC SYNDROME AMONG WORKERS OF DIFFERENT JOB CATEGORIES IN TAIWAN}

Chen-Chang Yang*, Hsin-Chien Wu*. National Yang-Ming University, Taipei city, Taiwan

\subsection{6/OEM-2019-EPI.235}

Background In Taiwan, the prevalence of metabolic syndrome among people over the age of 20 years is as high as $19.7 \%$. With the increase in age, the prevalence of metabolic syndrome is even higher, with the prevalence being more than 30 years among those aged $45-65$ years. Notably, very limited follow-up studies have examined the incidence and risk factors of metabolic syndrome among workers of different job categories in Taiwan.

Methods We conducted a retrospective follow-up study that included 6,284 Taiwanese subjects who had been working in the same job category for 5 years and who received periodic health checkups at a regional hospital from 2006 to 2017 to better understand the incidence rate and risk factors of metabolic syndrome in Taiwan. All participants' demographic data and health examination data were then analyzed. Metabolic syndrome was diagnosed according to the criteria proposd by 